\section{Sekundärprophylaxe nach Dissektion der Zervikalarterien}

Antikoagulation oder Plättchenhemmung? Diese Studie vergleicht die Antikoagulation mit Warfarin mit der Plättchenhemmung durch ASS - und bestätigt, dass es keine klare Evidenz für eine Bevorzugung einer der Methoden gibt.

$\mathrm{N}$ ach zervikalen Dissektionen kommt es insbesondere bei jüngeren Menschen zu Schlaganfällen. Welche Sekundärprävention ist hier die beste? In älteren Leitlinien und in der gängigen Praxis der meisten Einrichtungen wird für ein halbes Jahr die Antikoagulation empfohlen, danach sollte dauerhaft eine Plättchenhemmung etwa mit Acetylsalicylsäure (ASS) durchgeführt werden.

In diese dringend erwartete Studie wurden Patienten in Großbritannien und Australien eingeschlossen, die eine symptomatische Dissektion der Zervikalarterien entwickelt hatten. Diese Patienten wurden randomisiert über drei Monate entweder mit einer Antikoagu- lation oder mit einer Plättchenhemmung behandelt, wobei kein festes Protokoll vorgegeben war. Endpunkt war ein Tod oder Schlaganfall, der bei drei Patienten mit Plättchenhemmung und bei einem Patienten mit Antikoagulation auftrat. Der Unterschied war nicht signifikant. Interessant war auch, dass $10 \%$ der Patienten mit einer Dissektion initial mit eiren, ohne dass Probleme aufgetreten waren. Weiter wurde eine Analyse angeschlossen, in der durch Bildgebung das erneute Auftreten von Dissektionen evaluiert wurde. Auch hierbei ergab sich kein signifikanter Unterschied zwischen den beiden Behandlungsarmen. ner Thrombolyse behandelt worden wa-

\title{
Sekundärprophylaxe nach lakunären Hirninfarkten
}

\section{Bei den meisten Patienten scheint eine einfache Plättchenhemmung zur Prävention auszureichen - auf evidenzbasierte seriöse Studien dazu muss man noch warten.}

O bwohl lakunäre Infarkte etwa $25 \%$ aller ischämischen Hirninfarkte ausmachen, ist wenig über den Pathomechanismus und über die geeignete Sekundärprophylaxe bekannt. In den aktuellen Leitlinien wird eine Sekundärprävention mit einem Plättchenhemmer empfohlen, obwohl es unwahrscheinlich ist, dass eine Plättchenaggregation der Hauptmechanismus bei dieser Art von Hirninfarkten ist. Die meisten Daten stammen aus Subgruppenanalysen von großen Schlaganfallstudien und nicht aus Studien gezielt nur mit einer solchen Population.

In dieser Studie wurden 17 randomisierte Therapiestudien mit 42.234 Patienten einer Metaanalyse unterzogen. Für den Endpunkt Schlaganfall gab es je eine Studie mit Ticlopidin und Acetylsalicylsäure (ASS) versus Placebo. Hier ergab sich ein relatives Risiko von 0,77 , das statistisch signifikant war. Für den ischämischen Hirninfarkt gab es eine Studie mit ASS und eine mit Cilostazol. Hier ergab sich ein relatives Risiko von 0,48 , das ebenfalls signifikant war. Für den kombinierten Endpunkt Schlaganfall, Myokardinfarkt und Tod gab es eine Studie mit ASS sowie eine Studie mit ASS oder Dipyridamol. Hier ergab sich eine Risikoreduktion von 0,89 , die statistisch nicht signifikant war. Wurden Kombinationstherapien mit ASS verglichen, ergab sich für den ischämischen Schlaganfall ein relatives Risiko von 0,80 , was statistisch nicht signifikant war, und für den kombinierten End-

\section{Kommentar}

Diese Studie ist die erste randomisierte Studie, die eine Plättchenhemmung und eine Antikoagulation zur Sekundärprophylaxe bei Patienten mit einer Dissektion der Zervikalarterien vergleicht. Bislang gab es nur retrospektive Fallserien. Die Studie bestätigt, dass es keine klare Evidenz für eine Bevorzugung einer der beiden Methoden gibt. Man muss hierbei jedoch berücksichtigen, dass die Fallzahl sehr klein war und dass nicht auf Äquivalenz getestet werden konnte. Ein weiterer Aspekt ist, dass diese Studie eine Antikoagulation mit Warfarin durchgeführt hat. Ob bei einer Behandlung mit den neuen Antikoagulanzien ein anderes Ergebnis beobachtet worden wäre, kann nur spekuliert werden. Auf jeden Fall muss diese Studie reproduziert werden, bevor das Ergebnis Eingang in Leitlinien finden kann.

Prof. Dr. med. Dr. phil. Stefan Evers

CADISS trial investigators, Markus HS et al. Antiplatelet treatment compared with anticoagulation treatment for cervical artery dissection (CADISS): a randomised trial. Lancet Neurol 2015; 14 : $361-7$

punkt von 0,95 , ebenfalls ohne statistische Signifikanz.

\section{Kommentar}

Diese Metaanalyse zeigt, dass keine seriösen Studien vorliegen, die einen Hinweis auf die beste Sekundärprävention nach einem lakunären Hirninfarkt geben. Eine einfache Plättchenhemmung scheint bei der Mehrzahl der Patienten zu einer Prävention beizutragen, allerdings gilt dies wiederum für embolische Infarkte und nicht für erneute lakunäre Infarkte. Außerdem zeigt diese Metaanalyse, dass eine doppelte Plättchenhemmung in den meisten Fällen nicht besser wirksam ist als eine Monotherapie. Es bleibt somit bei der nur wenig evidenzbasierten Empfehlung, bei lakunären Hirninfarkten eine einfache Plättchenhemmung durchzuführen und auf weitere Studien zur besten Strategie nach lakunären Hirninfarkten zu warten.

Prof. Dr. med. Dr. phil. Stefan Evers

Kwok CS et al. Efficacy of antiplatelet therapy in secondary prevention following lacunar stroke: pooled analysis of randomized trials. Stroke 2015; 46: 1014-23 